\title{
Correcting SeaWinds Scatterometer Measurements for Atmospheric Attenuation
}

\author{
R. K. Moore, N. Kambhammettu, and S. Song \\ University of Kansas, Radar Systems and Remote Sensing Laboratory \\ 2291 Irving Hill Road, Lawrence, KS 66045-2969, USA \\ TEL: 913/864-4836 , FAX: 913/864-7789 , E-mail: rmoore@eecs.ukans.edu
}

\begin{abstract}
An attenuation-correction algorithm for the SeaWinds instrument uses the AMSR brightness temperature to find the attenuation experienced by the scatterometer signal. The algorithm uses the concept of excess temperature $T_{e x}$ to evaluate the attenuation. The excess temperature $T_{e x}$ is the difference between the measured brightness temperature and the temperature of the emitting surface. The algorithm uses an iterative scheme. Simulation results show obvious improvement on the scatterometer measurements.
\end{abstract}

\section{INTRODUCTION}

The wind vector on the ocean surface can be determined from measurements of radar backscatter of the ocean by using a spaceborne scatterometer [1]. The global coverage and frequent sampling give the spaceborne scatterometer advantages over other measurements [2]. However, the signal measured by the scatterometer is representative from the sea surface only when dense clouds and precipitation are absent. When heavy clouds and precipitation are present, the signal received at the scatterometer is reduced because of the attenuation of clouds and rain. For accurate determination of the sea surface wind vectors we need to estimate this reduction and make correction in the wind retrieval process.

Using radiometric measurements to make correction on the scatterometric data by combination was presented in [3]. A correction process was developed to evaluate the atmospheric attenuation from SMMR brightness temperature for the Seasat satellite scatterometer. The results showed the correction appeared to improve the accuracy of wind vector determination. However, the non-coincident large footprints of the two instruments negated this improvement in tight storms.

The SeaWinds scatterometer instrument will fly in 1999 as a part of the EOS program on the Japanese ADEOS-II. The scatterometer is a Ku-Band radar. It has antenna beams at two elevation angles giving a conical scan (concentric circles on the surface). The conical design represents a change from the fixed fan-beam antennas of previous spaceborne scatterometers (SeaSat and ERS-1). The on-board Advanced Microwave Scanning Radiometer (AMSR) works at multiple frequencies and also uses a conical scan. The concept of excess temperature is used to develop the correction algorithm for the scatterometer measurements by using the AMSR data. The AMSR and SeaWinds scans are not identical, but the smaller footprints than those on Seasat make comparison more reliable. The AMSR data must be collocated [5] to match the location of the scatterometer measurements.

\section{PRINCIPLES OF THE CORRECTION ALGORITHM}

When clouds and rain are present, the scatterometer signal will undergo a two-way attenuation. If we use $L$ to denote the one way attenuation, then the $\sigma^{0}$ measured by the scatterometer will be $\sigma^{\mathrm{m}}$ and

$$
\sigma_{m}=L^{2} \sigma^{0}=\sigma^{0} e^{-2 \alpha}
$$

where $\alpha$ is the attenuation factor in nepers. When clouds and rain are present, the radiometer measurements will be

$$
T_{m}=L\left(T_{s}+T_{r}\right)+T_{a t m}
$$

where $T_{s}$ is the sea-surface contribution and $T_{r}$ is the contribution from the sky emissions reflected by the sea surface. The last term $T_{a t m}$ is the contribution from the clouds and rain. The attenuation is denoted by $\mathrm{L}$, as in (1).

The excess temperature is defined as

$$
T_{e x}=T_{m}-T_{s}
$$

From (2), we know that if the surface temperature is known, the excess temperature will depend on $L, T_{a t m}$ and $T_{r}$. The $T_{r}$ is small comparing to $T_{a t m}$ and can be negligible. Thus the $\mathrm{T}_{e x}$ will be determined by the attenuation and the cloud and rain contribution. The relation between attenuation and excess temperature may be expressed by the cubic relation [4]

$$
\alpha(d B)=A T_{e x}+B T_{e x}^{2}+C T_{e x}^{3}
$$

From (2)-(4), an iterative process can be established to estimate the attenuation $L$ or $\alpha$, denoted by $L_{\text {est }}$ or $\alpha_{\text {est }}$. Using this factor, we can correct the scatterometer measured $\sigma^{0}$ by simply inverting equation (1)

$$
\sigma_{c}=\sigma_{m} e^{2 \alpha_{t x x}}
$$

However, the sea surface brightness temperature $T_{s}$ is needed when we calculate the excess temperature $T_{\text {ex }}$ by using (2) and (3). This can be obtain by using the following model [1] 

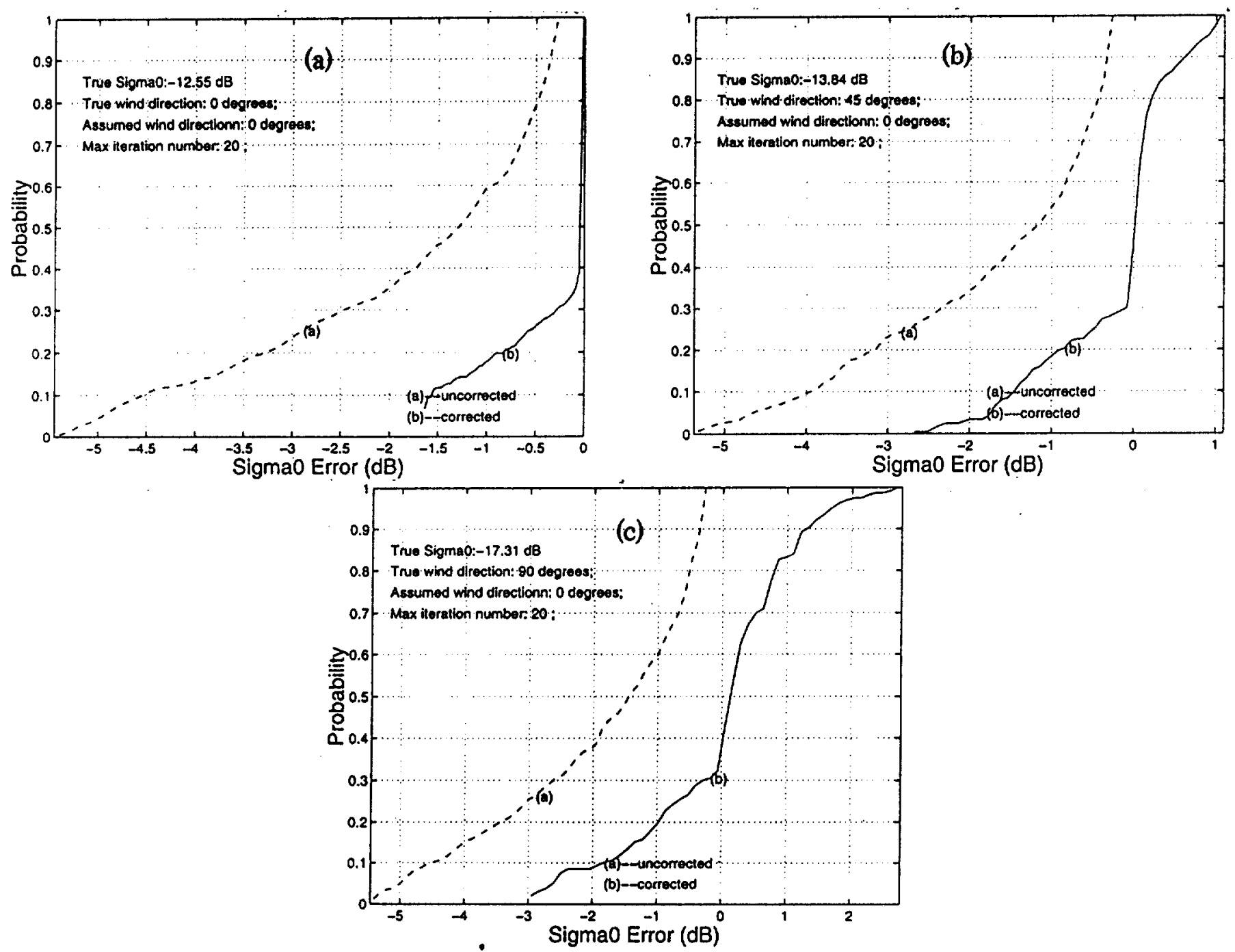

Fig. 1. Examples of error corrections for stratiform rain with different assumptions for wind. (a) Same direction as true wind, (b) $45^{\circ}$ from true wind, (c) $90^{\circ}$ from true wind.

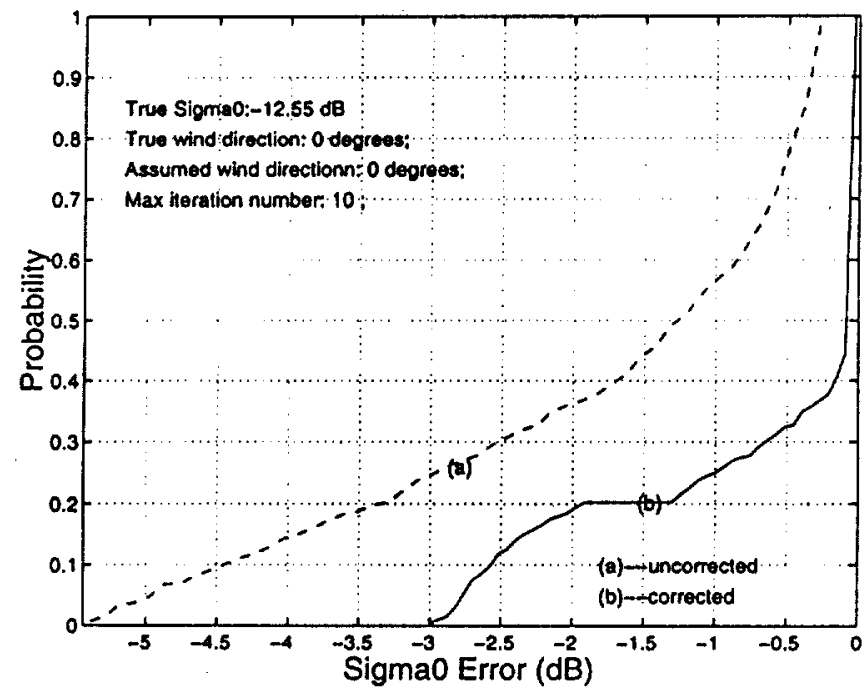

Fig. 2. Example of error correction for convective cells. Four cells in region with $r_{0}=7 \mathrm{~km}$. Note errors are small because much of footprint has little or no rain. 


$$
T_{s}=82+1.06 U
$$

where $U$ is the sea-surface wind speed, which can be estimated by the scatterometer-measured $\sigma^{\circ}$ using the SASS model function

$$
U_{e s t}=10 \frac{\sigma_{c}(d B)-10 G}{10 \mathrm{H}}
$$

where $\sigma^{\mathrm{c}}$ is from (5). G and $\mathrm{H}$ are constants of the model function that depend on the incident angle and the wind direction.

From above we can see how an iterative process can estimate the attenuation and correct the measured $\sigma^{0}$. However, in calculating (7), we need to know the wind direction. There are three possible solutions for this problem:

1. Assuming upwind direction;

2. Obtaining the wind direction using the SeaWinds aliasremoval algorithm;

3. Using the previous-cell wind direction.

The second solution has two difficulties. One is that the alias-removal Seawinds algorithm is quite complex and is not suitable for iteration. The other difficulty is the alias-removal SeaWinds algorithm uses a maximum-likelihood method and it needs to combine multi-point measurements of $\sigma^{0}$ to get the wind direction. However, we cannot obtain the accurate wind direction before correction is performed. So we have to use the first or the third solution. Obviously, the third solution is preferred as long as the wind-retrieval method can get accurate wind direction after correction. Simulation results for these two methods are given in the next section.

\section{SIMULATION AND RESULTS}

In the correction algorithm described above, we assumed the radiometer data used to estimate the attenuation is located at the same point as the scatterometer measurement. Actually, the measured points on the sea surface for the two instruments overlap but are not coincident. We need to match the scatterometer data with the proper radiometer data. A triangle method [5] is used to do the collocation in simulation. That is, for one scatterometer-measured $\sigma^{0}$, the nearest three points of AMSR measurements ( $\mathrm{Tb}$ ) will be collocated to this $\sigma^{0}$. Interpolation or weighting techniques are used to get the $T_{b}$ at the $\sigma^{0}$ point depending on whether the $\sigma^{0}$ point is inside or outside the triangle formed by three $T_{b}$ points.

In simulation, the cloud and rain distribution for convective storms is characterized by each storm having an exponential excess temperature distribution $T_{p} \exp \left(-r / r_{0}\right)$, where $T_{p}$ is the peak temperature at the storm center and $r$ is the distance from the center. The fall-off rate that determines the storm size is $\mathrm{r}_{0}$. For simulation, the number of storms is set and the peaks and centers of storms are randomly distributed. We ran the correction algorithm 400 times for different cloud and rain distributions to get the statistical results on the correction. Fig. 1 shows the error probability for corrected and uncorrected results for uniform rain throughout the region, as in a stratiform system, and shows the effect of wrong assumptions of direction. For Fig. 1(a) the direction assumed is correct; for $1(b)$, it is off by $45^{\circ}$, and for $1(\mathrm{c})$ it is off by $90^{\circ}$. Use of a direction from the preceding wind-vector cell should be well within $45^{\circ}$, and therefore acceptable. Arbitrary assumptions could be $90^{\circ}$ off, and the correction poorer. Fig. 2 shows a convective-storm situation with four storms, each with fall-off rate $r_{0}=7$. Even the uncorrected errors are small here, and the correction does improve the results.

\section{CONCLUSIONS}

A correction algorithm for the SeaWinds scatterometer, using AMSR estimates of attenuation, was presented. Simulated results show obvious improvement. Best results will be obtained if wind direction from the previous windvector cell can be used. Errors can be large with extensive rain unless corrected, but for small convective cells the errors are not large even before correction.

\section{REFERENCES}

[1] F. T. Ulaby, R. K. Moore, and A. K. Fung, Microwave Remote Sensing, vol. III, Artech House, Inc., Norwood MA, 1985.

[2] F. M. Nader, et al., "Spaceborne radar measurement of wind velocity over ocean-An overview of the NSCAT scatterometer system," Proc. IEEE, vol. 79, pp.850-866, 1991.

[3] R. K. Moore, A.H. Chaudhry, and I.J. Birrer, "Errors in scatterometer-radiometer wind measurement due to rain", IEEE J. Oceanic Eng., vol. OE-8, pp. 37-49, 1983.

[4] G. J. Dome, Oceanic Wind Vector Determination Using SeaSat Satellite, D. Eng. Thesis, Univ, of Kansas, Lawrence, 1980.

[5] SeaWinds Algorithm Review, JPL, Oct, 1995, unpublished. 